International Journal of Pure and Applied Mathematics

Volume 85 No. 2 2013, 193-202

ISSN: 1311-8080 (printed version); ISSN: 1314-3395 (on-line version)

url: http://www.ijpam.eu

doi: http://dx.doi.org/10.12732/ijpam.v85i2.1

ijpam.eu

\title{
MAJORIZATION FOR CERTAIN CLASSES OF ANALYTIC FUNCTIONS USING HURWITZ LERCH ZETA FUNCTION
}

\author{
K. Thilagavathi ${ }^{1}$, G. Murugusundaramoorthy ${ }^{2} \S$ \\ ${ }^{1,2}$ School of advanced Sciences \\ VIT University \\ Vellore, 632014, INDIA
}

\begin{abstract}
In the present paper, we investigate the majorization problems for functions belonging to the class $J_{s, b}^{p, n} f(z)$ is considered .Moreover we pointout some new or known consequences of our main result.
\end{abstract}

AMS Subject Classification: 30C45

Key Words: analytic functions, multivalent functions, starlikeness, convexity, Hadamard product, subordination, majorization, Hurwitz-Lerch zeta function

\section{Introduction}

Let $A_{p}(n)$ be the class of functions which are analytic and p-valent in the unit disk $U=\{z \in C:|z|<1\}$ of the form

$$
f(z)=z^{p}+\sum_{k=n}^{\infty} a_{p+k} z^{p+k}(p, n \in N=\{1,2, \ldots . .\}
$$

For $g(z) \in A_{p}(n)$ given by

$$
g(z)=z^{p}+\sum_{k=n}^{\infty} b_{k+p} z^{k+p}
$$

the Hadamard product of $f(z)$ and $g(z)$ is denoted by

Received: August 17, 2012

(C) 2013 Academic Publications, Ltd. url: www.acadpubl.eu

${ }^{\S}$ Correspondence author 


$$
(f * g)(z)=z^{p}+\sum_{k=n}^{\infty} a_{k+p} b_{k+p} z^{k+p}=(g * f)(z)
$$

The following we recall a general Hurwitz-Lerch Zeta function $\Phi(z, s, a)$ defined by (see [16])

$$
\Phi(z, s, a):=\sum_{k=0}^{\infty} \frac{z^{k}}{(k+a)^{s}}
$$

$\left(a \in \mathcal{C}\left\{\mathcal{Z}_{0}^{-}\right\} ; s \in \mathcal{C}, \Re(s)>1\right.$ and $\left.|z|=1\right)$ where, as usual, $\mathcal{Z}_{0}^{-}:=\mathcal{Z} \backslash\{\mathcal{N}\}$ $(\mathcal{Z}:=\{0, \pm 1, \pm 2, \pm 3, \ldots\} ; \mathcal{N}:=\{1,2,3, \ldots\})$. Several interesting properties and characteristics of the Hurwitz-Lerch Zeta function $\Phi(z, s, a)$ can be found in the recent investigations by Choi and Srivastava [3], Ferreira and Lopez [4], Garg et al. [5], Lin and Srivastava [7], Lin et al. [8], and others.

For the class of analytic functions denote by $\mathcal{A}$ consisting of functions of the form $f(z)=z+\sum_{k=2}^{\infty} a_{k} z^{k}$ Srivastava and Attiya [15] (see also Raducanu and Srivastava [13] ) introduced and investigated the linear operator:

$$
\mathcal{J}_{s, b}: \mathcal{A} \rightarrow \mathcal{A}
$$

defined in terms of the Hadamard product (or convolution) by

$$
\mathcal{J}_{s, b} f(z)=\mathcal{G}_{s, b} * f(z)
$$

$\left(z \in U ; b \in \mathcal{C} \backslash\left\{\mathcal{Z}_{0}^{-}\right\} ; \mu \in \mathcal{C} ; f \in \mathcal{A}\right)$, where, for convenience,

$$
G_{s, b}(z):=(1+b)^{s}\left[\Phi(z, s, b)-b^{-s}\right] \quad(z \in U) .
$$

It is easy to observe from (given earlier by [13]) (1), (5) and (6)that

$$
\mathcal{J}_{s, b} f(z)=z+\sum_{k=2}^{\infty}\left(\frac{1+b}{k+b}\right)^{s} a_{k} z^{k}
$$

Motivated essentially by the above-mentioned Srivastava-Attiya operator, we define the operator

$$
\mathcal{J}_{s, b}^{p, n}(f): \mathcal{A}_{p}(n) \rightarrow \mathcal{A}_{p}(n)
$$

which is defined as

$$
\mathcal{J}_{s, b}^{p, n} f(z)=z^{p}+\sum_{k=1}^{\infty} C_{b}^{s}(p, n) a_{p+k} z^{p+k} \quad\left(z \in U ; f(z) \in \mathcal{A}_{p}\right)
$$


where

$$
C_{b}^{s}(k, p)=\left(\frac{p+b}{k+p+b}\right)^{s}
$$

and (throughout this paper unless otherwise mentioned) the parameters $s, b$, are constrained as

$$
b \in \mathcal{C} \backslash\left\{\mathcal{Z}_{0}^{-}\right\} ; s \in \mathcal{C} \text { and } p, \in N .
$$

It is easily verified from (8)

$$
z\left(\mathcal{J}_{s+1, b}^{p, n} f\right)^{\prime}(z)=(p+b) \mathcal{J}_{s, b}^{p, n} f(z)-b \mathcal{J}_{s+1, b}^{p, n} f(z)
$$

For two analytic functions $f, g \in A_{p}$ we say that $f$ is subordinate to $g$ written $f(z) \prec g(z)$ if there exists a schwarz function $\omega(z)$ which (by definition) is analytic in $U$ with $\omega(0)=0$ and $|\omega(z)|<1$ for all $z \in U$, such that $f(z)=$ $g(\omega(z)), z \in U$.Furthermore, if the function $g(z)$ is univalent in $U$, then we have the following equivalence

$$
f(z) \prec g(z) \Longleftrightarrow f(0)=g(0) \operatorname{and} f(U) \subset g(U)
$$

If $f(z)$ and $g(z)$ are analytic functions in $U$,then due to MacGregor [9] we may say that $f(z)$ is majorized by $g(z)$ in $U$ and written as

$$
f(z)<<g(z)(z \in U)
$$

if there exists a function $\phi(z)$,analytic in $U$, such that

$$
|\phi(z)|<1 \text { and } f(z)=\phi(z) g(z)(z \in U)
$$

It is noted that the notation of majorization(12) is closely related to the concept of quasi-subordination between analytic functions in $U$ which was considered earlier by $[1,2]$,

on the other hand,investigated several majorization problems involving a number of subclasses of anlytic functions in $U$. In present sequel to the work of Altintas etal[2] we propose to investigate the corresponding majorization problem associated with the class of multivalent functions based on Srivastava-Attitya operator as defined below.

Definition 1. A function $f(z) \in A_{p}(n)$ and suppose that $g(z) \in J_{s, b}^{p, n}(\gamma)$ of p-valent functions of complex order $\gamma \neq 0 i n U$ if and only if

$$
\begin{gathered}
\operatorname{Re}\left(1+\frac{1}{\gamma}\left(z \frac{J_{s+1, b}^{p, n} f^{(q+1)}(z)}{J_{s+1, b}^{p, n} f^{(q)}(z)}-p+q\right)\right)>0 \\
\left(z \in U ; p \in N ; q \in N_{0} ; \gamma \in C-\{0\} ;|2 \gamma-(p+q)| \leq(p+q)\right)
\end{gathered}
$$


Motivated by earlier works of $[6,10,12,14]$ in this paper we investigate majorization problems for the function class $J_{s, b}^{p, n}(\gamma)$ of $p$-valently stalike functions of complex order $\gamma \neq 0$ in open unit $\operatorname{disc} U$.

\section{Main Result}

Theorem 1. A function $f(z) \in A_{p}(n)$ and suppose that $g(z) \in J_{s, b}^{p, n}(\gamma)$ if $\left[J_{s+1, b}^{p, n} f(z)\right]^{(q)}$ is majorized by $\left[J_{s+1, b}^{p, n} g(z)\right]^{(q)}$ in $U$ then

$$
\left|\left[J_{s, b}^{p, n} f(z)\right]^{(q)}\right| \leq\left|\left[J_{s, b}^{p, n} g(z)\right]^{(q)}\right|\left(|z| \leq r_{0}\right)
$$

where $r_{0}$ is given by

$$
r_{0}=r_{0}(p, b, \gamma)=\frac{L-\sqrt{L^{2}-4(p+q)|2 \gamma-(p+q)|}}{2|2 \gamma-(p+q)|}
$$

where $L=(p+b)+|2 \gamma-(p+b)| ; p \in N ; \gamma \in C-(0)$

Proof. since $g(z) \in J_{s, b}^{p, n}(\gamma)$ we find from ,if

$$
h(z)=1+\frac{1}{\gamma}\left(\frac{\left[J_{s+1, b}^{p, n} g(z)\right]^{(q+1)}}{\left[J_{s+1, b}^{p, n} g(z)\right]^{(q)}}-p+q\right)
$$

then $\Re\{h(z)\}>0(z \in U)$ and

$$
h(z)=\frac{1+\omega(z)}{1-\omega(z)}(\omega \in A)
$$

where

$$
\omega(z)=c_{1} z+c_{2} z^{2}+\ldots
$$

and $A$ denotes the well known class of bounded analytic functions in $U$ and satisfies the conditions

$$
\omega(0)=0, \text { and }|\omega(z)| \leq|z|(z \in U)
$$

making use of (17) and(18) we get

$$
\frac{\left[J_{s+1, b}^{p, n} g(z)\right]^{(q+1)}}{\left[J_{s+1, b}^{p, n} g(z)\right]^{(q)}}=\frac{p+q+(2 \gamma-p+q) \omega(z)}{1-\omega(z)}
$$


In view of equation(10)

$$
\left|\left[J_{s+1, b}^{p, n} g(z)\right]^{(q)}\right| \leq \frac{(1+|z|)(p+b)}{p+b-(2 \gamma-p+b)|z|}\left|\left[J_{s, b}^{p, n} g(z)\right]^{(q)}\right|
$$

Since $\left[J_{s+1, b}^{p, n} f(z)\right]^{(q)}$ is majorized by $\left[J_{s, b}^{p, n} g(z)\right]^{(q)}$ in $U$ then we have

$$
\left[J_{s+1, b}^{p, n} f(z)\right]^{(q)}=\phi(z)\left[J_{s+1, b}^{p, n} g(z)\right]^{(q)}
$$

Differentiating with respect to $\mathrm{z}$ and then multiplying $\mathrm{z}$ we get

$$
z\left(\left[J_{s+1, b}^{p, n} f(z)\right]^{(q+1)}=z \phi^{\prime}(z)\left[J_{s+1, b}^{p, n} g(z)\right]^{(q)}+z \phi(z)\left[J_{s+1, b}^{p, n} g(z)\right]^{(q+1)}\right.
$$

Using (10) in the above equation we get

$$
(p+b)\left[J_{s, b}^{p, n} f(z)\right]^{(q)}=\left(\frac{z}{p+b}\right)\left(\varphi^{\prime}(z)\right)\left[J_{s+1, b}^{p, n} g(z)\right]^{(q)}+\varphi(z)\left[J_{s, b}^{p, n} g(z)\right]^{(q)}
$$

Noting that the Schwarz function $\phi(z)$ satisfies

$$
\left|\phi^{\prime}(z)\right| \leq \frac{1-|\phi(z)|^{2}}{1-|z|^{2}}
$$

and using (22) and(26) in (25) we have

$$
\begin{aligned}
& \left|\left[J_{s, b}^{p, n} f(z)\right]^{(q)}\right| \\
& \quad \leq\left\{|\phi(z)|+\frac{|z|\left(1-|\phi(z)|^{2}\right)}{(1-|z|)} \frac{1}{(p+b)-|(2 \gamma-(p+b))||z|}\right\}\left|\left[J_{s, b}^{p, n} g(z)\right]^{(q)}\right|
\end{aligned}
$$

setting $|z|=r$ and $|\phi(z)|=\rho(0 \leq \rho \leq 1)$

$$
\left|\left[J_{s, b}^{p, n} f(z)\right]^{(q)}\right| \leq \frac{\psi(\rho)}{(1-r)\{(p+b)-|(2 \gamma-(p+b))| r\}}\left|\left[J_{s, b}^{p, n} g(z)\right]^{(q)}\right|
$$

where

$$
\psi(\rho)=-\rho^{2} r+\rho(1-r)\{(p+b)-|2 \gamma-(p+b)| r\}+r
$$

takes its maximum value at $\rho=1$ with $r_{0}=r_{0}(p, b, \gamma)$ given by Furthermore,if

$0 \leq \sigma \leq r_{0}=r_{0}(p, b, \gamma)$, the function $\varphi(\rho)$ defined by

$$
\varphi(\rho)=-\rho^{2} r+\rho(1-r)\{(p+b)-|2 \gamma-(p+b)| r\}+r
$$

is an increasing function on $(0 \leq \rho \leq 1)$ so that

$$
\varphi(\rho) \leq \varphi(1)=(1-r)((p+b)-|(2 \gamma-(p+b))| r)
$$

$(0 \leq \rho \leq 1), 0 \leq \sigma \leq r_{0}=r_{0}(p, b, \gamma)$ then setting $\rho=1$ in we conclude that holds true for $|z| \leq r_{0}(p, b, \gamma)$. This completes the proof of Theorem 1 . 
Corollary 2. Let the function $f(z) \in A_{p}$ and $g(z) \in J_{s, b}^{1, n}(\gamma)$ If $\left[J_{s+1, b}^{1, n} f(z)\right]$ is majorized by $\left[J_{s+1, b}^{1, n} g(z)\right]$ in $U$, then

$$
\left|\left[J_{s, b}^{1, n} f(z)\right]\right| \leq\left|\left[J_{s, b}^{1, n} g(z)\right]\right|\left(|z| \leq r_{1}\right),
$$

where

$$
r_{1}=\frac{Q \pm \sqrt{Q^{2}-4(1+q)|2 \gamma-(1+q)|}}{2|2 \gamma-(1+q)|}
$$

where

$$
Q=(1+b)+|2 \gamma-(1+b)|
$$

Putting $q=0$ and

$$
\gamma=\left(1-\frac{\alpha}{p}\right) \cos \lambda e^{-i \lambda}\left(|\lambda|<\frac{\pi}{2} ; 0 \leq \alpha \leq p\right)
$$

in Theorem 1 we have the following corollory

Corollary 3. Let the function $f(z) \in A_{p}$ and $g(z) \in J_{s, b}^{p, n}(\alpha)\left(|\lambda|<\frac{\pi}{2}\right)$. If

$$
\left|J_{s, b}^{p, n} f(z)\right| \leq\left|J_{s, b}^{p, n} g(z)\right|\left(|z| \leq r_{2}\right)
$$

$r_{2}=r_{2}(p, b, \lambda)$ is given by

$$
r_{2}=\frac{\delta \pm \sqrt{\delta^{2}-4(p+b)\left|2\left(1-\frac{\alpha}{p}\right) \cos \lambda e^{-i \lambda}-(b+p)\right|}}{2\left|2\left(1-\frac{\alpha}{p}\right) \cos \lambda e^{-i \lambda}-(p+b)\right|}
$$

where

$$
\delta=(p+b)+\left|2\left(1-\frac{\alpha}{p}\right) \cos \lambda e^{-i \lambda}-(p+b)\right|
$$

putting $m=0$ in corollary 2 we have the following corollary:

The proof of our next result is essentially based upon the following lemma, for the class of starlike and convex functions of complex order $\gamma$ considered and studied by Nasar[11].

Lemma 1. If $f \in C(\gamma)$, the class of convex functions of order $\gamma$ where $(\gamma \in C\{0\})$, then $f \in S\left(\frac{1}{2} \gamma\right)$, that is $C(\gamma) \subset S\left(\frac{1}{2} \gamma\right)$ the class of starlike functions of order $\frac{\gamma}{2}$.

Theorem 4. Let the function $f(z) \in A_{p}$ and $g(z) \in C_{s, b}^{p, n}$ if $J_{s+1, b}^{p, n} f(z)$ is majorized by $J_{s+1, b}^{p, n} g(z)$ in $U$ then

$$
\left|\left[J_{s, b}^{p, n} f(z)\right]^{(q)}\right| \leq\left|\left[J_{s, b}^{p, n} g(z)\right]^{(q)}\right|\left(|z| \leq r_{4}\right)
$$


where $r_{4}$ is given by

$$
r_{4}=\frac{T-\sqrt{T^{2}-4(p+q)|\gamma-(p+q)|}}{2|\gamma-(p+q)|}
$$

where

$$
T=(p+b)+|\gamma-(p+b)|
$$

The proof of our next result is essentially based upon the following lemmas due to Altintas[1].

Lemma 2. If the function $h(z)$ of the form $h(z)=1-\sum_{k=1}^{\infty} c_{k} z^{k}$ be in the class $R(\lambda, \gamma)$ if it satisfies the condition

$$
\Re\left(h^{\prime}(z)+\lambda z h^{\prime}(z)\right)>\alpha
$$

then

$$
\sum_{k=1}^{\infty} c_{k} \leq \frac{\gamma}{1+\Re(\lambda)}
$$

Lemma 3. If the function $h(z)$ of the form $h(z)=1-\sum_{k=1}^{\infty} c_{k} z^{k}$ be in the class $R(\lambda, \gamma)$ if it satisfies the condition

$$
\Re\left(h^{\prime}(z)+\lambda z h^{\prime}(z)\right)>\alpha
$$

then

$$
1-\frac{|\gamma|}{1+\Re(\lambda)}|z| \leq|h(z)| \leq 1+\frac{|\gamma|}{1+\Re(\lambda)}|z|,(z \in U)
$$

Finally we prove

Theorem 5. Let the function $f(z) \in A_{p}$ and $g(z) \in R(\lambda, \gamma)$ be analytic in $U$ and suppose that the function $g(z)$ is so normalised that it also satisfies the following inclusion property:

$$
\frac{\left[J_{s, b}^{p, n} g(z)\right]^{(q)}}{\left[J_{s+1, b}^{p, n} g(z)\right]^{(q)}} \in R(\lambda, \gamma)
$$

if $\left[J_{s, b}^{p, n} f(z)\right]^{(q)}$ is majorized by $\left[J_{s, b}^{p, n} g(z)\right]^{(q)}(z \in U)$, then

$$
\left|\left[J_{s, b}^{p, n} f(z)\right]^{(q)}\right| \leq\left|\left[J_{s, b}^{p, n} g(z)\right]^{(q)}\right|\left(|z| \leq r_{5}\right)
$$


where $r_{5}$ is given by

$$
r_{5}=r_{5}(\lambda, \gamma)
$$

is the smallest root of the equation

$$
|\gamma| r^{3}-\left(1+\Re(\lambda) r^{2}\right)-[2+|\gamma|+2 \Re(\lambda)] r+1+\Re(\lambda)=0
$$

Proof. For an appropriately normalised analytic function $g(z)$ satisfying the inclution property we find from the assertion of Lemma 3 that

$$
\left|\frac{\left[J_{s, b}^{p, n} g(z)\right]^{(q)}}{\left[J_{s+1, b}^{p, n} g(z)\right]^{(q)}}\right| \geq 1-\frac{|\gamma|}{1+\Re(\lambda)} r(|z|=r ; 0<r<1)
$$

or equivalently that

$$
\left|\left[J_{s+1, b}^{p, n} g(z)\right]^{(q)}\right| \leq \frac{1+\Re(\lambda)}{1+\Re(\lambda)-|\gamma| r}\left|\left[J_{s, b}^{p, n} g(z)\right]^{(q)}\right|(|z|=r ; 0<r<1)
$$

Since

$$
\left[J_{s+1, b}^{p, n} f(z)\right]^{(q)} \ll\left[J_{s+1, b}^{p, n} g(z)\right]^{(q)}(z \in U)
$$

there exists an analytic function $\omega$ such that

$$
\left[J_{s+1, b}^{p, n} f(z)\right]^{(q)}=\omega(z)\left[J_{s+1, b}^{p, n} g(z)\right]^{(q)}
$$

and $|\omega(z)|<1$ Thus in view of and just as in the proof of Theorem 1, we have $|\omega(z)| \leq \frac{1-|\omega(z)|^{2}}{1-|z|^{2}}(z \in U)$ and

$$
\left[J_{s, b}^{p, n} f(z)\right]^{(q)} \leq\left(|\omega(z)|+\frac{1-|\omega(z)|^{2}}{1-r^{2}} \frac{1+\Re(\lambda)}{1+\Re(\lambda)-|\gamma| r}\right)\left[J_{s, b}^{p, n} g(z)\right]^{(q)}
$$

Where we have set $|\omega(z)|=\rho$ and the function $\theta(\rho)$ defined by

$$
\theta(\rho)=\{1+\Re(\lambda)\}+\left(1-r^{2}\right) 1+\Re(\lambda)-|\gamma| r \rho-\{1+\Re(\lambda)\} \rho^{2}(0 \leq \rho \leq 1)
$$

takes on its maximum value at $\rho=1$ with $r=r_{5}(\lambda, \gamma)$ given by Moreover if $0 \leq \eta \leq r_{5}(\lambda, \gamma)$ where $r_{5}(\lambda, \gamma)$ is the root of the cubic equation such that $0<r_{5}(\lambda, \gamma)<1$ then the function $\vartheta(\rho)$ defined by

$$
\vartheta(\rho)=\{1+\Re(\lambda)\}+\left(1-\eta^{2}\right) 1+\Re(\lambda)-|\gamma| \eta \rho-\{1+\Re(\lambda)\} \rho^{2}(0 \leq \rho \leq 1)
$$

is seen to be increasing function on the interval $0 \leq \rho \leq 1$, so that

$$
\vartheta(\rho)=\theta(1)=\left(1-\eta^{2}\right) 1+\Re(\lambda)-|\gamma| \eta\left(0 \leq \rho \leq 1 ; 0 \leq \eta \leq r_{5}(\lambda, \gamma)\right.
$$

consequently,upon setting $\rho=1$ in ,we complete the proof of Theorem 3 . 


\section{References}

[1] O. Altintas, S. Owa, Majorization and quasi-subordinations for certain analytic functions, Proc. Japan Acad. Ser. A Math. Sci., 68 (1992), 181185.

[2] O. Altintas, O. Ozkan, H.M. Srivastava, Majorization by starlike functions of complex order, Complex Variable Theory Appl., 46 (2001), 207-218.

[3] J. Choi, H.M. Srivastava, Certain families of series associated with the Hurwitz-Lerch Zeta function, Appl. Math. Comput., 170 (2005), 399-409.

[4] C. Ferreira, J.L. López, Asymptotic expansions of the Hurwitz-Lerch zeta function, J. Math. Anal. Appl., 298 (2004), 210-224.

[5] M. Garg, K. Jain, H.M. Srivastava, Some relationships between the generalized Apostol-Bernoulli polynomials and Hurwitz-Lerch zeta functions, Integral Transform. Spec. Funct., 17 (2006), 803-815.

[6] S.P. Goyal, P. Goswami, Majorization for certain classes of analytic functions defined by fractional derivatives, Appl. Math. Lett. (2009).

[7] S.-D. Lin, H.M. Srivastava, Some families of the Hurwitz-Lerch Zeta functions and associated fractional derivative and other integral representations, Appl. Math. Comput., 154 (2004), 725-733.

[8] S.-D. Lin, H. M. Srivastava, P.-Y. Wang, Some espansion formulas for a class of generalized Hurwitz-Lerch Zeta functions, Integral Transform. Spec. Funct., 17 (2006), 817-827.

[9] T.H. MacGreogor, Majorization by univalent functions, Duke Math. J., 34 (1967), 95-102.

[10] A.O. Mostafa, M.K. Aouf, Majorization for certain class of multivalent functions defined by differential operator, Applied Mathematics Letters.

[11] M.A. Nasr, M.K. Aouf, Starlike function of complex order, J. Natur. Sci. Math., 25 (1985), 1-12.

[12] Pranay Goswami, M.K Aouf, Majorization properties for certain class of analytic functions using the salagean operator, Applied Mathematics Letters, 23 (2010), 1351-1354. 
[13] D. Raducanu, H.M. Srivastava, A new class of analytic functions defined by means of a convolution operator involving the Hurwitz-Lerch Zeta function, Integral Transform. Spec. Funct., 18 (2007), 933-943.

[14] C. Selvaraj, K.A. Selvakumaran, Majorization Problems for certain classes of analytic functions, International Mathematical Forum, 6, No. 6 (2011), 289-294.

[15] H.M. Srivastava, A.A. Attiya, An integral operator associated with the Hurwitz-Lerch Zeta function and differential subordination, Integral Transform. Spec. Funct., 18 (2007), 207-216.

[16] H.M. Srivastava, J. Choi, Series Associated with the Zeta and Related Functions, Dordrecht-Boston-London, Kluwer Academic Publishers (2001). 